\title{
Diethylstilbestrol Dipropionate
}

National Cancer Institute

\section{Source}

National Cancer Institute. Diethylstilbestrol Dipropionate. NCI Thesaurus. Code C74557.

The dipropionate ester of a synthetic, nonsteroidal form of estrogen. A well-known teratogen and carcinogen, diethylstilbestrol inhibits the hypothalamic-pituitary-gonadal axis, thereby blocking the testicular synthesis of testosterone, lowering plasma testosterone, and inducing a chemical castration. 